\title{
Operative volume and outcomes of cerebrovascular neurosurgery in children
}

\author{
Kimon Bekelis, MD, ${ }^{1,2}$ Ian D. Connolly, MS, ${ }^{3,4}$ Huy M. Do, MD, ${ }^{3}$ and Omar Choudhri, MD ${ }^{4}$ \\ ${ }^{1}$ Section of Neurosurgery, Dartmouth-Hitchcock Medical Center; ${ }^{2}$ Dartmouth Institute for Health Policy and Clinical Practice, \\ Lebanon, New Hampshire; ${ }^{3}$ Departments of Radiology and Neurosurgery, Stanford University School of Medicine, Palo Alto; and \\ ${ }^{4}$ Department of Neurosurgery, University of California, San Francisco, California
}

\begin{abstract}
OBJECTIVE The impact of procedural volume on the outcomes of cerebrovascular surgery in children has not been determined. In this study, the authors investigated the association of operative volume on the outcomes of cerebrovascular neurosurgery in pediatric patients.
\end{abstract}

METHODS The authors performed a cohort study of all pediatric patients who underwent a cerebrovascular procedure between 2003 and 2012 and were registered in the Kids' Inpatient Database (KID). To control for confounding, the authors used multivariable regression models, propensity-score conditioning, and mixed-effects analysis to account for clustering at the hospital level.

RESULTS During the study period, 1875 pediatric patients in the KID underwent cerebrovascular neurosurgery and met the inclusion criteria for the study; 204 patients (10.9\%) underwent aneurysm clipping, $446(23.8 \%)$ underwent coil insertion for an aneurysm, 827 (44.1\%) underwent craniotomy for arteriovenous malformation resection, and 398 (21.2\%) underwent bypass surgery for moyamoya disease. Mixed-effects multivariable regression analysis revealed that higher procedural volume was associated with fewer inpatient deaths (OR $0.58 ; 95 \% \mathrm{Cl} 0.40-0.85$ ), a lower rate of discharges to a facility (OR $0.87 ; 95 \% \mathrm{Cl} 0.82-0.92)$, and shorter length of stay (adjusted difference $-0.22 ; 95 \% \mathrm{Cl}-0.32$ to -0.12 ). The results in propensity-adjusted multivariable models were robust.

CONCLUSIONS In a national all-payer cohort of pediatric patients who underwent a cerebrovascular procedure, the authors found that higher procedural volume was associated with fewer deaths, a lower rate of discharges to a facility, and decreased lengths of stay. Regionalization initiatives should include directing children with such rare pathologies to a center of excellence.

http://thejns.org/doi/abs/10.3171/2016.5.PEDS16137

KEY WORDS cerebrovascular neurosurgery; pediatrics; procedural volume; Kids' Inpatient Database; vascular disorders

$\mathrm{C}$ EREBROVASCULAR neurosurgery is technically demanding, and its practice is typically limited to surgeons with particular expertise. ${ }^{1}$ Most vascular pathology is encountered in adults. Although rare, pediatric cerebrovascular disease poses challenges related to not only the pathology but also patient age and the specifics of clinical management in this population. Pediatric patients have significantly smaller blood vessels, a lower atherosclerotic burden, a higher frequency of syndromic pathology with multiple lesions, and diseases that are extremely rare in adults, such as moyamoya disease and vein of Galen malformations. ${ }^{7-9}$ Although several investigators have found an association between procedural volume and outcomes for several surgical procedures, $,, 6,14$ this association has not been shown in pediatric patients who have undergone cerebrovascular neurosurgery.

ABBREVIATIONS AVM = arteriovenous malformation; ICD-9-CM = International Classification of Diseases, 9th Revision, Clinical Modification; KID = Kids' Inpatient Database; LOS = length(s) of stay.

SUBMITTED March 6, 2016. ACCEPTED May 25, 2016.

INCLUDE WHEN CITING Published online August 5, 2016; DOI: 10.3171/2016.5.PEDS16137. 
Previous studies have examined the relationship between volume and outcomes in adults who were undergoing interventions for several cerebrovascular pathologies and have demonstrated conflicting results. The results of single-center series ${ }^{13,15}$ and international investigations ${ }^{16}$ have supported the idea that surgeon volume does not correlate with outcome. These results have limited generalization, given their inherent selection bias. However, national studies in the United States have found evidence to the contrary..$^{3,10,19}$ To our knowledge, no previous study has investigated the association between pediatric procedural volume and outcomes of cerebrovascular neurosurgery in a national comprehensive cohort of pediatric patients.

We performed a cohort study of pediatric patients who were registered in the Kids' Inpatient Database (KID) to investigate the association of procedural volume with inpatient death, length of stay (LOS), and discharge to a facility after cerebrovascular procedures. We used a battery of approaches, including regression adjustment, propensity score conditioning, and mixed-effects analysis, to control for confounding and account for clustering at the hospital level.

\section{Methods}

\section{Kids' Inpatient Database}

The KID is part of a family of databases and software tools developed for the Healthcare Cost and Utilization Project. It is the largest publicly available all-payer pediatric inpatient care database in the United States. It contains data from approximately 3 million pediatric discharges each year. All patients who underwent a cerebrovascular procedure and were registered in the KID between 2003 and 2012 were included in this analysis. For these years, the KID contains patient-level details for more than 4100 US community hospitals (nonfederal) as coded from billing records. More information about the KID is available at https://www.hcup-us.ahrq.gov/kidoverview.jsp.

\section{Cohort Definition}

We used International Classification of Diseases, 9th Revision, Clinical Modification (ICD-9-CM) codes to identify patients with an unruptured or ruptured cerebral aneurysm (diagnosis code 437.3 or 430 , respectively) who underwent aneurysm clipping (procedure code 39.51 ) or coil therapy (procedure code $39.72,39.75,39.76$, or 39.79 ), patients who underwent craniotomy (procedure code $01.51,01.52,01.53$, or 01.59) for arteriovenous malformation (AVM) (diagnosis code 747.81) resection, and patients who underwent bypass surgery (procedure code 39.28 ) for moyamoya disease (diagnosis code 437.5) between 2003 and 2012.

\section{Outcome Variables}

The primary outcome was inpatient death. Secondary outcomes were LOS for the initial hospitalization and the rate of discharges to a facility.

\section{Exposure Variables}

The primary exposure variable was the annual pro- cedural volume of the treating neurosurgeon, which was treated as a continuous variable. For parts of the analysis, it was treated as a categorical variable with 4 distinct categories.

Treatment modality (clipping, coiling, craniotomy for AVM, or bypass surgery for moyamoya disease) was included in the analysis. Covariates used for risk adjustment were age, sex, and race (African American, Hispanic, Asian, Caucasian, or other). The comorbidities used for risk adjustment were diabetes mellitus, smoking, hypertension, congestive heart failure, history of stroke, drug abuse, obesity, chronic renal failure, history of cancer, and coagulopathy. In the analyses, we considered only variables that were present as part of the patient's preadmission comorbidity profile.

\section{Statistical Analysis}

To investigate the association of outcomes with procedural volume, we used several methods to address confounding that have been used before in the literature. ${ }^{3}$ For inpatient death and discharge to a facility, we initially used multivariable logistic regression, including all the covariates listed above, and the surgeon's pediatric cerebrovascular case volume as a continuous variable. To account for clustering of observations at the hospital level, we used mixed-effects models with hospital identification as a random-effects variable. For LOS, we used multiple linear regression models. In the sensitivity analysis, we repeated this approach after logarithmic transformation of LOS. The observed associations did not change and therefore are not reported further. In addition, we created quartiles of procedural volume and repeated these analyses to find a clinically interpretable estimate of the associations.

To further control for confounding for our binary outcomes, we used regression models with adjustment (stratification) by quantiles (we chose the number of quantiles to be 20) of propensity score. To derive the propensity for undergoing treatment by a high-volume neurosurgeon, we developed a prediction model using logistic regression based on the covariates described above. High volume was defined as practice volume equal to or higher than the average procedural volume of all physicians. To account for clustering of observations at the hospital level, we used mixed-effects models with hospital identification as a random-effects variable.

In addition, in the sensitivity analysis, we repeated these analyses for only the surgeon's pediatric cerebrovascular case volume in the year before the intervention in question. Last, in prespecified subgroup analyses, we examined the differences in outcomes for different procedures (clipping, coiling, craniotomy for AVM resection, and bypass surgery for moyamoya disease) and controlled for all covariates listed previously, including case volume and the presence of subarachnoid hemorrhage. Our results were remarkably robust in all of these sensitivity analyses. The directions of the observed associations were identical in these different iterations, and therefore, those results are not reported here.

Given that we had 1875 patients and an average pro- 
TABLE 1. Patient characteristics*

\begin{tabular}{|c|c|c|c|}
\hline Characteristic & $\begin{array}{c}\text { All } \\
\text { Patients } \\
(n=1875)\end{array}$ & $\begin{array}{l}\text { Underwent } \\
\text { Surgery by } \\
\text { Higher-Than- } \\
\text { Average-Vol } \\
\text { Surgeon } \\
(n=1251)\end{array}$ & $\begin{array}{l}\text { Underwent } \\
\text { Surgery by } \\
\text { Lower-Than- } \\
\text { Average-Vol } \\
\text { Surgeon } \\
(\mathrm{n}=624)\end{array}$ \\
\hline Age (yrs) & $12.5 \pm 5.8$ & $13.1 \pm 5.7$ & $11.2 \pm 5.8$ \\
\hline \multicolumn{4}{|l|}{ Race } \\
\hline Caucasian & $988(52.7)$ & $666(53.2)$ & $320(51.3)$ \\
\hline African American & $257(13.7)$ & $187(14.9)$ & $69(11.1)$ \\
\hline Hispanic & $375(20.0)$ & $254(20.3)$ & $121(19.4)$ \\
\hline Asian & $124(6.6)$ & $56(4.5)$ & $68(10.9)$ \\
\hline Native American & $19(1.0)$ & $15(1.2)$ & $4(0.6)$ \\
\hline Other & $112(6.0)$ & $73(6.0)$ & $42(6.7)$ \\
\hline Gender (female) & $936(49.9)$ & $602(48.1)$ & $334(53.5)$ \\
\hline \multicolumn{4}{|l|}{ Comorbidity } \\
\hline Anemia & $103(5.5)$ & $79(6.3)$ & $24(4.0)$ \\
\hline Congestive heart failure $†$ & $11(0.6)$ & - & - \\
\hline Chronic lung disease & $131(7.0)$ & $90(7.2)$ & $42(6.7)$ \\
\hline Coagulopathy & $49(2.6)$ & $39(3.1)$ & - \\
\hline Diabetes mellitus $†$ & - & - & - \\
\hline Drug abuse & $26(1.4)$ & $23(1.8)$ & - \\
\hline Hypertension & $159(8.5)$ & $110(8.8)$ & $49(7.9)$ \\
\hline Obesity & $34(1.8)$ & $25(2.0)$ & - \\
\hline Neurological deficit & $36(1.9)$ & $31(2.5)$ & - \\
\hline Renal failure $\dagger$ & - & - & - \\
\hline Weight loss & $19(1.0)$ & $16(1.3)$ & - \\
\hline Cancer† & - & - & - \\
\hline
\end{tabular}

cedural volume of 4 (SD 4.7), we had an $80 \%$ power to detect a difference in mortality rates as small as $6.5 \%$ between the 2 groups at an $\alpha$ level of 0.05 . Patients with missing data $3 \%$ missing race data were excluded from further analysis. All probability values were the result of 2 -sided tests. Stata 13 (StataCorp) was used for the statistical analyses.

\section{Results}

\section{Patient Characteristics}

Between 2003 and 2012, 1875 pediatric patients (average age 12.5 years, $49.9 \%$ female) in the KID underwent a cerebrovascular procedure and met the inclusion criteria for the study; 204 patients (10.9\%) underwent cerebral aneurysm clipping, 446 (23.8\%) underwent coil insertion for cerebral aneurysms, 827 (44.1\%) underwent craniotomy for AVM resection, and 398 (21.2\%) underwent bypass surgery for moyamoya disease. The respective distributions of exposure variables between patients treated by above- and below-average-volume proceduralists are listed in Table 1. Procedural volumes at the surgeon level ranged from
TABLE 2. Correlation of procedural volume with primary and secondary outcomes*

\begin{tabular}{|c|c|c|}
\hline $\begin{array}{l}\text { Primary or Secondary Outcomes } \\
\text { According to Model }\end{array}$ & Value & $\begin{array}{c}\mathrm{p} \\
\text { Value }\end{array}$ \\
\hline \multicolumn{3}{|l|}{ Inpatient death $\ddagger(\mathrm{OR}[95 \% \mathrm{CI}])$} \\
\hline Crude & $0.66(0.51-0.86)$ & $<0.001$ \\
\hline Multivariable regression & $0.58(0.40-0.85)$ & $<0.001$ \\
\hline Propensity score adjustment† & $0.27(0.08-0.91)$ & $<0.001$ \\
\hline \multicolumn{3}{|l|}{ Discharge to a facility $\ddagger$ (OR [95\% Cl]) } \\
\hline Crude & $0.86(0.82-0.90)$ & $<0.001$ \\
\hline Multivariable regression & $0.87(0.82-0.92)$ & $<0.001$ \\
\hline Propensity score adjustment† & $0.55(0.39-0.77)$ & $<0.001$ \\
\hline \multicolumn{3}{|l|}{ LOS§ (adjusted difference [95\% CI]) } \\
\hline Crude & $-0.32(-0.43$ to -0.22$)$ & $<0.001$ \\
\hline Multivariable regression & $-0.22(-0.32$ to -0.12$)$ & $<0.001$ \\
\hline
\end{tabular}

* Procedural volume was treated as a continuous variable for all analyses except the propensity-score-adjusted model, in which the procedural volume was treated as a dichotomous variable with high volume defined as volume equal to or higher than the average procedural volume of the entire cohort. $\dagger$ Propensity-score calculation was based on age, race, sex, anemia, and comorbidities (i.e., congestive heart failure, chronic lung disease, coagulopathy, diabetes mellitus, drug abuse, hypertension, obesity, neurologic deficit, renal failure, weight loss, cancer).

$\ddagger$ Analyses based on logistic regression model with treatment hospital as a random-effects variable.

$\S$ Based on linear regression model with treatment hospital as a randomeffects variable.

1 to 23 (average 4) pediatric cerebrovascular procedures per year.

\section{Inpatient Death}

Among pediatric patients undergoing cerebrovascular procedures, 39 deaths $(2.1 \%)$ were recorded. As shown in Table 2, higher procedural volume was associated with fewer inpatient deaths (OR 0.66; 95\% CI 0.51-0.86) in the unadjusted analysis. Similarly, in a multivariable logistic regression model (Table 2), higher procedural volume was associated with a lower inpatient mortality rate (OR 0.58 ; 95\% CI 0.40-0.85). This effect persisted after propensityscore adjustment (OR 0.27; 95\% CI 0.08-0.91).

\section{Length of Stay}

Among pediatric patients undergoing cerebrovascular procedures, the median LOS was 5 days (interquartile range 8 days). As shown in Table 2, higher procedural volume was associated with shorter LOS (adjusted difference $-0.32 ; 95 \%$ CI -0.43 to -0.22 ) in the unadjusted analysis. Similarly, in a multivariable logistic regression model (Table 2), increased procedural volume was associated with shorter LOS (adjusted difference -0.22 ; $95 \%$ CI -0.32 to $-0.12)$.

\section{Discharge to a Facility}

Among pediatric patients undergoing cerebrovascular procedures, $306(16.3 \%)$ were discharged to a facility. As 
TABLE 3. Correlation of categories of procedural volume with primary and secondary outcomes*

\begin{tabular}{|c|c|c|}
\hline $\begin{array}{l}\text { Outcomes According to Annual } \\
\text { No. of Procedures }\end{array}$ & OR $(95 \% \mathrm{Cl})$ & $\mathrm{p}$ Value \\
\hline \multicolumn{3}{|l|}{ Inpatient death $\dagger$} \\
\hline $1-5$ & 1 (reference) & \\
\hline $6-10$ & $0.50(0.18-1.41)$ & 0.191 \\
\hline $10-20$ & $0.21(0.06-0.82)$ & 0.024 \\
\hline$>20$ & $0.09(0.02-0.54)$ & $<0.001$ \\
\hline \multicolumn{3}{|l|}{ Discharge to a facility $\dagger$} \\
\hline $1-5$ & 1 (reference) & \\
\hline $6-10$ & $0.84(0.56-1.27)$ & 0.416 \\
\hline $10-20$ & $0.70(0.55-0.85)$ & $<0.001$ \\
\hline$>20$ & $0.35(0.22-0.55)$ & $<0.001$ \\
\hline \multicolumn{3}{|l|}{ LOS $\ddagger$ (adjusted difference [95\% CI]) } \\
\hline $1-5$ & 1 (reference) & \\
\hline $6-10$ & $-0.9(-1.8$ to -0.1$)$ & $<0.001$ \\
\hline $10-20$ & $-1.2(-1.9$ to -0.5$)$ & $<0.001$ \\
\hline$>20$ & $-2.6(-3.8$ to -1.3$)$ & $<0.001$ \\
\hline
\end{tabular}

* Procedural volume was treated as a continuous variable for all analyses except the propensity-score-adjusted model, in which the procedural volume was treated as a dichotomous variable with high volume defined as volume equal to or higher than the average procedural volume of the entire cohort. † Analyses based on logistic regression model with treatment hospital as a random-effects variable.

\pm Based on linear regression model with treatment hospital as a randomeffects variable.

shown in Table 2, higher procedural volume was associated with a lower rate of discharge to a facility (OR 0.86; 95\% CI 0.82-0.90) in the unadjusted analysis. Similarly, in a multivariable logistic regression model (Table 2), higher procedural volume was associated with a lower rate of discharge to a facility (OR 0.87 ; $95 \%$ CI $0.82-0.92$ ). This effect persisted after propensity-score adjustment (OR 0.55; 95\% CI 0.39-0.77). Table 3 shows the respective associations across categories of procedural volume.

\section{Discussion}

Using a national comprehensive all-payer cohort of pediatric patients who underwent cerebrovascular neurosurgery, we identified an association between increased procedural volume and fewer inpatient deaths, shorter LOS, and a lower rate of discharges to a facility. Our results were consistent across several advanced observational techniques, which we used to control for confounders and clustering at the hospital level. Subgroup analyses confirmed these associations for cerebral aneurysm clipping and coil insertion, AVM resection, and bypass surgery for moyamoya disease. Treatment of patients with these conditions can be demanding, given the challenges of the pathologies and the characteristics of this age group. Little attention has been paid to the impact of procedural volume of treating neurosurgeons on the outcomes of cerebrovascular neurosurgery in pediatric patients.

Previous data on the association of case volume and outcomes for cerebral aneurysm treatment for adults have been conflicting. Several small studies ${ }^{13,15,16}$ have supported the idea that surgeon volume does not correlate with outcomes. However, national US-based studies have had different results..$^{10,19}$ In a study of the Nationwide Inpatient Sample that used patients with an unruptured aneurysm who underwent coil insertion, higher hospital and surgeon volumes were associated with decreased morbidity but had no significant effect on the mortality rate. ${ }^{18}$ In a cohort limited to New York state, Zacharia et al. ${ }^{26}$ found that a higher hospital volume was associated with a lower discharge-to-rehabilitation rate. Bekelis et al. ${ }^{3}$ found that an increasing volume of coil therapy was associated with improved outcomes in patients with an unruptured aneurysm, with a ceiling effect at 20 cases.

The outcomes of cerebrovascular neurosurgery in children have been examined in previous studies. ${ }^{7-9,17,20,21,25} \mathrm{Be}-$ cause the need for such surgery is rare in pediatric patients, most investigations used retrospective analyses of singlecenter experiences. ${ }^{7-9,17,20,21,25}$ Their results, therefore, have limited generalization. The lack of control for clustering and the limited use of multivariable methods also restrict the scope of these investigations. In one of the most recent series, Sanai et al. ${ }^{23}$ summarized the 30-year experience of treating pediatric cerebral aneurysms in a single referral center. They were able to report on only 32 patients, $78 \%$ of whom had a good neurological recovery. The common denominator of these experiences is that pediatric cerebral aneurysms are more commonly giant or fusiform, posterior circulation based, and present with mass effect rather than subarachnoid hemorrhage. ${ }^{7-9,17,20,21,25}$ In addition, mycotic aneurysms are observed more frequently in children than in adults (10\% vs $2 \%-3 \%$, respectively, of all cerebral aneurysms). ${ }^{7}$ Analyses of the treatment of AVMs and vein of Galen malformations have not been focused specifically on children but have found synergy of the multimodal treatment options available. ${ }^{8,9}$ No previous investigation has focused on the impact of procedural volume on outcomes for this patient population.

In our study of a broad range of cerebrovascular pathologies, we intentionally addressed these limitations. First, we created a national cohort of pediatric patients in which global practice in this age group was represented. To our knowledge, we have analyzed real-world pediatric cerebrovascular practices for the first time. Second, we used advanced observational techniques, including propensity stratification and mixed-effects analysis, to control for confounding. The results were consistent across techniques, which supports the validity of the observed associations.

The results of our analyses call for centralization of care for pediatric cerebrovascular neurosurgery in concert with other regionalization incentives in medicine..$^{26,27}$ Previous investigations have noticed a shift in the care of cerebral aneurysms to low-volume centers for adults, ${ }^{12}$ which is likely secondary to the widespread use of endovascular treatments and their application in smaller institutions. ${ }^{12,26,27}$ We found incrementally improved outcomes for surgeons with increasing volumes. Results of our current analysis indicate that a procedural volume of more than 20 pediatric cerebrovascular interventions is 
associated with the lowest chance of an adverse outcome. Appropriate volume cutoffs should be studied further by surgeons, payers, administrators, and policy makers to effectively inform change and affect referral patterns.

Our study has several limitations that are common to research performed with information from administrative databases. First, there is always a possibility of residual confounding. However, this possibility is minimized with the various advanced techniques we used for risk adjustment. Second, coding inaccuracies undoubtedly occur and might have affected our estimates. Previous studies used these codes to identify patients undergoing cerebrovascular interventions. ${ }^{1,2,4,10,11,18,26,27}$ ICD-9-CM codes cannot differentiate AVMs from cavernous malformations or other vascular malformations. Therefore, the ICD-9-CM codes for vascular malformations represent all such lesions and have no specificity. However, several reports have shown that coding for cerebral aneurysms and cerebrovascular disease has had nearly perfect association with medical record review. 22,24

Third, the KID does not provide any clinical information on the size, structure, or location of pathology, which are important factors in cerebrovascular neurosurgery. However, because neurosurgeons with busier practices are expected to see the most complicated cases, this lack of information would bias our results toward the null and would lessen the observed associations. Fourth, we were lacking long-term data on our patients. Quality metrics (i.e., modified Rankin Scale) were also not available through this database. In the future, these measures can be investigated using long-term registries, which are currently in development (e.g., see http://www.neuropoint.org/ NPA\%20N2QOD.aspx). Last, despite the use of advanced techniques, we cannot establish causality based on results from our cohort.

\section{Conclusions}

The impact of operative volume on the outcomes of cerebrovascular procedures in children has not been determined. In a national all-payer cohort of pediatric patients who underwent a cerebrovascular procedure, we found that higher procedural volume was associated with fewer deaths, a lower rate of discharge to a facility, and shorter LOS. Regionalization initiatives should direct patients with such rare pathologies to a center of excellence.

\section{References}

1. Bekelis K, Goodney RP, Dzebisashvili N, Goodman DC, Bronner KK: Variation in the care of surgical conditions: cerebral aneurysms, in Goodney PR, Dzebisashvili N, Goodman DC, et al (eds): Variations in the Care of Surgical Conditions: A Dartmouth Atlas of Health Care Series. Lebanon, NH: The Dartmouth Institute, 2014

2. Bekelis K, Gottlieb D, Bovis G, Su Y, Tjoumakaris S, Jabbour P, et al: Unruptured cerebral aneurysm clipping: association of combined open and endovascular expertise with outcomes. J Neurointerv Surg [epub ahead of print], 2015

3. Bekelis K, Gottlieb D, Labropoulos N, Su Y, Tjoumakaris S,
Jabbour P, et al: The impact of hybrid neurosurgeons on the outcomes of endovascular coiling for unruptured cerebral aneurysms. J Neurosurg [epub ahead of print February 26, 2016. DOI: $10.3171 / 2015.11 . J N S 151725]$

4. Bekelis K, Missios S, MacKenzie TA: Continuity of care and 30-day readmission for patients evaluated in the emergency room after cerebral aneurysm treatment. J Neurointerv Surg [epub ahead of print], 2016

5. Birkmeyer JD, Siewers AE, Finlayson EV, Stukel TA, Lucas FL, Batista I, et al: Hospital volume and surgical mortality in the United States. N Engl J Med 346:1128-1137, 2002

6. Birkmeyer JD, Stukel TA, Siewers AE, Goodney PP, Wennberg DE, Lucas FL: Surgeon volume and operative mortality in the United States. N Engl J Med 349:21172127, 2003

7. Blount JP, Oakes WJ, Tubbs RS, Humphreys RP: History of surgery for cerebrovascular disease in children. Part I. Intracranial arterial aneurysms. Neurosurg Focus 20(6):E9, 2006

8. Blount JP, Oakes WJ, Tubbs RS, Humphreys RP: History of surgery for cerebrovascular disease in children. Part II. Vein of Galen malformations. Neurosurg Focus 20(6):E10, 2006

9. Blount JP, Oakes WJ, Tubbs RS, Humphreys RP: History of surgery for cerebrovascular disease in children. Part III. Arteriovenous malformations. Neurosurg Focus 20(6):E11, 2006

10. Brinjikji W, Kallmes DF, Lanzino G, Cloft HJ:

Hospitalization costs for endovascular and surgical treatment of ruptured aneurysms in the United States are substantially higher than Medicare payments. AJNR Am J Neuroradiol 33:1037-1040, 2012

11. Brinjikji W, Kallmes DF, Lanzino G, Cloft HJ: Hospitalization costs for endovascular and surgical treatment of unruptured cerebral aneurysms in the United States are substantially higher than Medicare payments. AJNR Am J Neuroradiol 33:49-51, 2012

12. Brinjikji W, Lanzino G, Kallmes DF, Cloft HJ: Cerebral aneurysm treatment is beginning to shift to low volume centers. J Neurointerv Surg 6:349-352, 2014

13. Chang TR, Kowalski RG, Carhuapoma JR, Tamargo RJ, Naval NS: Impact of case volume on aneurysmal subarachnoid hemorrhage outcomes. J Crit Care 30:469472,2015

14. Finks JF, Osborne NH, Birkmeyer JD: Trends in hospital volume and operative mortality for high-risk surgery. $\mathbf{N}$ Engl J Med 364:2128-2137, 2011

15. Goldschlager T, Selvanathan S, Walker DG: Can a "novice" do aneurysm surgery? Surgical outcomes in a low-volume, non-subspecialised neurosurgical unit. J Clin Neurosci 14:1055-1061, 2007

16. Hattori N, Katayama Y, Abe T: Case volume does not correlate with outcome after cerebral aneurysm clipping: a nationwide study in Japan. Neurol Med Chir (Tokyo) 47:95-101, 2007

17. Herman JM, Rekate HL, Spetzler RF: Pediatric intracranial aneurysms: simple and complex cases. Pediatr Neurosurg 17:66-73, 1991-1992

18. Hoh BL, Rabinov JD, Pryor JC, Carter BS, Barker FG II: In-hospital morbidity and mortality after endovascular treatment of unruptured intracranial aneurysms in the United States, 1996-2000: effect of hospital and physician volume. AJNR Am J Neuroradiol 24:1409-1420, 2003

19. Johnston SC: Effect of endovascular services and hospital volume on cerebral aneurysm treatment outcomes. Stroke 31:111-117, 2000

20. Kalani MY, Elhadi AM, Ramey W, Nakaji P, Albuquerque FC, McDougall CG, et al: Revascularization and pediatric aneurysm surgery. J Neurosurg Pediatr 13:641-646, 2014 
21. Kim SK, Wang KC, Kim DG, Paek SH, Chung HT, Han $\mathrm{MH}$, et al: Clinical feature and outcome of pediatric cerebrovascular disease: a neurosurgical series. Childs Nerv Syst 16:421-428, 2000

22. Kokotailo RA, Hill MD: Coding of stroke and stroke risk factors using international classification of diseases, revisions 9 and 10. Stroke 36:1776-1781, 2005

23. Sanai N, Quinones-Hinojosa A, Gupta NM, Perry V, Sun PP, Wilson CB, et al: Pediatric intracranial aneurysms: durability of treatment following microsurgical and endovascular management. J Neurosurg 104 (2 Suppl):82-89, 2006

24. Tirschwell DL, Longstreth WTJ Jr: Validating administrative data in stroke research. Stroke 33:2465-2470, 2002

25. Vanaman MJ, Hervey-Jumper SL, Maher CO: Pediatric and inherited neurovascular diseases. Neurosurg Clin N Am 21:427-441, 2010

26. Zacharia BE, Bruce SS, Carpenter AM, Hickman ZL, Vaughan KA, Richards C, et al: Variability in outcome after elective cerebral aneurysm repair in high-volume academic medical centers. Stroke 45:1447-1452, 2014

27. Zacharia BE, Ducruet AF, Hickman ZL, Grobelny BT, Badjatia N, Mayer SA, et al: Technological advances in the management of unruptured intracranial aneurysms fail to improve outcome in New York state. Stroke 42:2844-2849, 2011

\section{Disclosures}

The authors report no conflict of interest concerning the materials or methods used in this study or the findings specified in this paper.

\section{Author Contributions}

Conception and design: Bekelis. Acquisition of data: Connolly, Do, Choudhri. Analysis and interpretation of data: Bekelis. Drafting the article: Bekelis. Critically revising the article: Connolly, Do, Choudhri. Reviewed submitted version of manuscript: Bekelis, Connolly. Approved the final version of the manuscript on behalf of all authors: Bekelis. Statistical analysis: Bekelis. Study supervision: Bekelis.

\section{Correspondence}

Kimon Bekelis, Section of Neurosurgery, Dartmouth-Hitchcock Medical Center, One Medical Center Dr., Lebanon, NH 03755. email: kbekelis@gmail.com. 